Systematic Review

\title{
Are Pain Beliefs, Cognitions, and Behaviors Influenced by Race, Ethnicity, and Culture in Patients with Chronic Musculoskeletal Pain: A Systematic Review
}

Ceren Orhan, $\mathrm{PhD}^{1,2}$, Eveline Van Looveren, $\mathrm{MSc}^{2,3}$, Barbara Cagnie, $\mathrm{PhD}^{2}$

Naziru Bashir Mukhtar, MSc' ${ }^{2}$ Dorine Lenoir, $\mathrm{MSc}^{2,3}$, and Mira Meeus, $\mathrm{PhD}^{2-4}$

From: ${ }^{1}$ Department of Physiotherapy and Rehabilitation, Hacettepe University, Turkey; ${ }^{2}$ Department of Rehabilitation

Sciences and Physiotherapy, Ghent University, Belgium; ${ }^{3}$ Pain in Motion international research group, www.paininmotion.be; ${ }^{4}$ Department of Rehabilitation Sciences and Physiotherapy, University of Antwerp, Belgium

Address Correspondence: Mira Meeus, PhD Ghent University, Department of Rehabilitation Sciences and Physiotherapy,

Corneel Heymanslaan 10, 9000 Ghent, Belgium.

E-mail: mira.meeus@ugent.be

Disclaimer: Lenoir and Meeus contributed equally to this manuscript. The first author (CO) was financially supported by the Scientific Researches Department of Hacettepe University during the study period in Ghent University (Project number: TBI2017-13866).

Manuscript received: 03-29-2018

Revised manuscript received: 05-29-2018

Accepted for publication: 06-01-2018

Free full manuscript: www.painphysicianjournal.com
Background: Chronic pain has been considered as a biopsychosocial condition in which cognitive and emotional factors as well as biological factors significantly affect perception of pain. Race, ethnicity and culture have a crucial impact on illness beliefs, health care preferences, help-seeking behaviors, and acceptance of medical interventions.

Objectives: The aim of the present study was to systematically review the current evidence regarding the racial, ethnic and cultural alterations and differences in pain beliefs, cognitions, and behaviors in patients with chronic musculoskeletal pain (MSKP).

Study Design: Systematic review.

Methods: This systematic review was conducted and reported in accordance with the Preferred Reporting Items for Systematic reviews and Meta-analyses guidelines (PRISMA). PubMed and Web of Science were searched. A first screening was conducted based on title and abstract of the articles. In the second screening, full-texts of the remaining articles were evaluated for the fulfilment of the inclusion criteria. The risk of bias was assessed with the modified Newcastle-Ottawa Scale.

Results: A total of 11 articles were included. The methodological quality of the included studies ranged from low to moderate. There is moderate evidence that African-Americans use more praying, hoping, and emotion-focused coping strategies than Caucasians. There is also preliminary evidence regarding the differences in some coping strategies such as distraction, catastrophizing, and problem-focused solving between African-Americans and Caucasians. Preliminary evidence exists regarding the differences in pain coping strategies between the US and Portugal; the US and Singapore; and among 4 French-speaking countries. It is found that Spanish patients with fibromyalgia (FM) have more negative illness perceptions than Dutch patients. There is preliminary evidence that Caucasians have higher self-efficacy than African-Americans. There is also preliminary evidence that New Zealanders have more internal health expectancies than patients from the US. Preliminary evidence is demonstrated that Caucasians with rheumatoid arthritis (RA) have more positive control beliefs than African-Americans. Lastly, there is preliminary evidence that patients from the US believe that they are more disabled, while Singaporeans interpret the pain more by a traditional biomedical perspective.

Limitations: Only 11 articles were included. The small number of articles, wide range of assessment methods, and substantial risk of bias in the included studies led the investigator to draw conclusions cautiously.

Conclusion: Preliminary to moderate evidence shows the differences in coping strategies, illness perceptions, self-efficacy, fear avoidance beliefs, locus of control, and pain attitudes in different populations. Further prospective and longitudinal studies using standard definitions for race, ethnicity or culture and valid questionnaires for each population are warranted to explore the racial, ethnic and cultural discrepancies in pain beliefs, cognitions, and behaviours.

Key words: Chronic pain, musculoskeletal pain, pain beliefs, pain cognitions, pain behaviors, race, ethnicity, culture

Pain Physician 2018: 21:541-558 
hronic pain has been defined as pain persisting longer than completion of a normal healing process and therefore, it has been considered to be a dysfunction of the acute warning mechanism of physiological nociception (1). In general practice, pain is interpreted as chronic when it lasts more than 3 months (2). Chronic musculoskeletal pain (MSKP) is one of the most common types of chronic pain and is considered as a major health problem (3-5). A large European study has revealed that $19 \%$ of the participants had chronic pain (6). According to the epidemiological studies, chronic MSKP is associated with an increase in age, female gender, non-Caucasian race, low socioeconomic status, and a high stress level $(5,7)$. Chronic pain has been considered as a biopsychosocial condition in which contextual, cognitive and emotional factors as well as biological factors significantly affect pain perception (8-11).

In the literature, many beliefs, cognitions, appraisals, behaviors, and thought processes about the nature of pain were identified including catastrophizing, pain-related anxiety and fear, locus of control, helplessness, hyper vigilance, self-efficacy, coping, readiness to change and acceptance (11-14). Although pain-related beliefs and cognitions sometimes might be adaptive by protecting the injured area in acute pain, transferring these pain beliefs from acute pain to chronic pain often results in fear of movement, increased disability, and greater pain $(11,15,16)$. Previous research showed that positive pain beliefs, cognitions, and behaviors were strongly associated with improved functional outcomes and better treatment adherence (17-20). It has been reported that the changes in pain-related beliefs seems to be associated with the changes in pain intensity, pain interference, and psychological functioning (2123). Therefore, it has been widely accepted that pain beliefs, cognitions, and behaviors are important factors and determinants for diagnosis, evaluation and treatment in patients suffering from chronic pain (17).

However, race, ethnicity, and culture have a crucial impact on these illness beliefs, health care preferences, help-seeking behaviors, and acceptance of medical interventions (24). The meaning of pain, the evaluation and interpretation of pain, and the consequent emotional and behavioural responses may be influenced by race, ethnicity, and culture (25). Furthermore, locus of control, cultural mistrust, religion, pain model and feelings may lead to variations in pain beliefs, cognitions, and behaviors (25). Over the past 2 decades, there is growing literature reporting the influence of cultural factors, race, and ethnicity on pain-related disparities across different populations (26-30). Literature investigating racial, ethnic, and cultural factors related to pain, have been focused on the pain epidemiology, pain-related outcomes such as clinical, experimental and psychological, assessment and treatment of pain, and health utilization (26-30). A significant proportion of the research revealed the differences between black/ African American, Hispanic/Latino, and non-Hispanic white populations in pain-related disparities (26).

On the other hand, a systematic review investigating the racial and ethnic disparities in pain management concluded that the major problem of the studies was the inconsistent and unclear use of the race and ethnicity terms (31). According to the previous studies, it has been emphasized that race, ethnicity, and culture have different meanings $(25,28,32,33)$. While race is usually used to distinguish groups of people based on the physical characteristics, ancestry and heredity $(25,26,28,32)$, the term ethnicity implies that groups of people share a common culture, language, traditions, religion, and behaviors as well as physical characteristics $(28,33,34)$. The concept of culture contains a unity of ideas, beliefs, values, and knowledge within a certain group of people (25), that sometimes is considered as a nation or national boundaries (33). However, these definitions are not universally accepted (26).

According to our knowledge, studies comparing pain beliefs, cognitions, and behaviors between different racial, ethnic, and cultural populations have not been systematically reviewed. Additionally, there is no clear overview of the influence of race, ethnicity and culture on pain-related beliefs, cognitions, and behaviors in patients with chronic MSKP. A previous meta-analysis investigated the racial differences in pain coping between African-Americans and Caucasians in both healthy individuals, and patients with chronic pain (35). The constructive knowledge about racial, ethnic, and cultural differences and alterations in pain beliefs, cognitions, and behaviors is essential for diagnosis, assessment and treatment of persistent pain. Therefore, the aim of the present study is to systematically review the current evidence regarding the racial, ethnic, and cultural alterations and differences in pain beliefs, cognitions, and behaviors of patients with chronic MSKP.

\section{Methods}

This systematic review was conducted and reported in accordance with the Preferred PRISMA guidelines (36). 
The protocol of the systematic review was registered on PROSPERO (Registration number: CRD42018088051).

\section{Research Question}

The research question was formulated using the Patient, measurement Instrument, Comparison, Outcome (PICO) approach. It was determined as: "What are the disparities in pain beliefs, cognitions, and behaviors ( $O$ = outcome) assessed with quantitative measurements ( $I$ = instrument) between different racial, ethnic, or cultural populations ( $C=$ comparison) in patients with chronic MSKP (P = patients)?"

\section{Information Sources and Search Strategy}

To identify relevant publications regarding the racial, ethnic, and cultural differences in pain beliefs, cognitions, and behaviors, PubMed (https://www.ncbi. nlm.nih.gov/pubmed) and Web of Science (https://apps. webofknowledge.com) were searched on January 3, 2018. The search strategy consisted of the combination of free text words and MeSH terms (only for searching PubMed). The complete search strategy in PubMed was: (chronic pain OR persistent pain) AND (race OR culture OR ethnicity OR migrant OR cultural OR ethnic OR racial OR latino OR latina OR american OR african OR white OR asian OR black OR caucasian OR hispanic) AND (pain AND (pain belief OR pain perception OR attitude OR self-efficacy OR fear-avoidance OR pain cognition OR catastrophi* OR "locus of control" OR anticipation OR behaviour OR coping OR acceptance OR kinesiophobia OR somatization OR vigilance)) AND (Humans[Mesh] AND adult[MeSH]).

\section{Eligibility Criteria}

To be included in this systematic review, studies had to meet the following eligibility criteria: 1) adults older than 18 years of age; 2) patients with chronic MSKP; 3 ) evaluation of the pain-related beliefs, cognitions, and behaviors such as self-efficacy, fear avoidance, catastrophization, locus of control, acceptance, coping, somatization, and vigilance; 4) comparison of disparities in pain-related beliefs and cognitions between different racial, ethnic, and cultural populations; 5) assessment of pain-related beliefs, cognitions, and behaviors with quantitative and self-reported measurements; 6) availability of full-text articles in English, Dutch or French; 7) case-control, cohort or cross-sectional study design. Exclusion criteria were: animal studies; study sample of children or adolescents ( $<18$ years of age); not examining the differences in pain beliefs, cognitions, and behaviors among different racial, ethnic, and cultural populations; assessing the pain beliefs, cognitions, and behaviors with interviews; not available full-texts; abstracts and posters.

\section{Study Selection}

The study selection consisted of 2 phases. First and second screening were performed by using an online tool named RAYYAN (https://rayyan.qcri.org) (37). First screening was conducted based on title and abstract of the articles that corresponded to the inclusion criteria. If an article did not meet the inclusion criteria, it was excluded. In the second screening, remaining full-text articles were evaluated once more for the fulfilment of the inclusion criteria. In addition, to find the eligible articles that were not identified by the search strategy, reference lists of the included articles were screened.

The second screening was performed in accordance with the inclusion and exclusion criteria by 2 independent researchers (CO and $\mathrm{DL}$ ) who are respectively post-doctoral researcher and PhD student working on chronic pain and who were trained in conducting a systematic review by a third researcher (MM). The study selection was supervised and disagreements were solved by the third researcher (MM) who is a professor in the domain of chronic pain.

\section{Risk of Bias Assessment in Individual Studies and Quality of Evidence}

Methodological quality of all included studies was evaluated by 2 independent researchers (CO and EV) who are respectively post-doctoral researcher and PhD student, currently working on chronic pain and they were trained in assessing risk of bias by the third researcher (MM). Discrepancies were solved by the decision of the third researcher (MM). The risk of bias was assessed with the modified Newcastle-Ottawa Scale (NOS), which has been recommended for case control studies (http://www.ohri.ca/programs/clinical_epidemiology/oxford.asp) (38,39). This checklist is also advised by the Cochrane Collaboration (www.cochrane.org). To determine the risk of bias, a star rating system was applied to 3 dimensions including selection of groups, comparability, and ascertainment and exposure (40). The definitions of cases (items 1 and 4) were rated based on the information about race, ethnicity, or culture regarding the birthplace, country in which the person is living, and parent's country of origin. An extra item (item 7) was added to the "Comparability dimension." Items 5, 6, and 7 evaluated whether the 
Table 1. Methodological quality for case-control studies.

\begin{tabular}{|c|c|c|c|c|c|c|c|c|c|c|c|c|c|c|c|}
\hline \multirow[b]{2}{*}{ Study } & \multicolumn{4}{|c|}{ Selection } & \multicolumn{3}{|c|}{ Comparability } & \multicolumn{3}{|c|}{ Exposure 1} & \multicolumn{3}{|c|}{ Exposure $2^{\mathrm{a}}$} & \multirow{2}{*}{$\begin{array}{c}\text { Total } \\
\text { score }(\%)\end{array}$} & \multirow{2}{*}{$\begin{array}{l}\text { Level of } \\
\text { evidence }\end{array}$} \\
\hline & 1 & 2 & 3 & 4 & 5 & 6 & 7 & 8 & 9 & 10 & 11 & 12 & 13 & & \\
\hline Allen et al (42) & - & - & - & - & - & + & + & + & - & + & + & - & + & $6^{\mathrm{b}}(46)$ & B \\
\hline Cano et al (44) & - & + & + & - & - & - & - & + & + & - & 1 & 1 & 1 & $4^{c}(40)$ & $\mathrm{B}$ \\
\hline Golightly et al (43) & - & - & - & - & - & + & - & + & - & + & + & - & + & $5^{\mathrm{b}}(38)$ & B \\
\hline Jones et al (47) & - & + & + & - & - & + & - & + & - & + & + & - & + & $7^{\mathrm{b}}(54)$ & B \\
\hline Jordan et al (48) & - & - & - & - & + & + & + & + & - & + & + & - & + & $7^{\mathrm{b}}(54)$ & B \\
\hline Ferreira-Valente et al (45) & - & - & - & - & - & - & - & + & - & + & + & - & + & $4^{\mathrm{b}}(31)$ & $\mathrm{B}$ \\
\hline Thong et al (49) & - & - & - & - & + & + & - & + & - & + & + & - & - & $5^{\mathrm{b}}(38)$ & B \\
\hline Genet et al (46) & - & - & - & - & - & - & + & + & - & + & + & - & + & $5^{\mathrm{b}}(38)$ & B \\
\hline Cho et al (50) & - & - & - & - & + & - & - & + & - & - & 1 & 1 & 1 & $2^{c}(20)$ & B \\
\hline Ruiz- Montero et al (51) & - & + & + & - & - & + & - & + & + & - & 1 & I & 1 & $5^{c}(50)$ & B \\
\hline Tait et al (52) & - & - & - & - & + & - & - & + & - & + & 1 & 1 & 1 & $3^{c}(30)$ & B \\
\hline
\end{tabular}

$1=$ Case definition (birthplace, country in which the person is living, and parent's country of origin ${ }^{*}$ ); $2=$ Representativeness of the cases (random sample $\left.{ }^{\star}\right) ; 3=$ Selection of controls (random sample $\left.{ }^{*}\right) ; 4=$ Definition of controls (birthplace, country in which the person is living, and parent's country of origin $\left.{ }^{*}\right) ; 5=$ Study controls for age ${ }^{*} ; 6=$ Study controls for gender $* 7=$ Study controls for pain duration $* 8=$ Same method of ascertainment for both groups $s^{*} ; 9=$ Ascertainment of exposure (valid questionnaire for each population ${ }^{\star}$ ); $10=$ Non-response rate (similar between groups $\left.^{*}\right) ; \mathrm{a}=$ The items of 11,12 , and 13 are similar to the items 8,9 , and 10 , respectively; $\mathrm{b}=$ Maximum score is $13 ; \mathrm{c}=$ Maximum score is 10 .

Table 2. Level of evidence, according to the 2005 classification system of the Dutch Institute for Healthcare Improvement CBO (www.cbo.nl).

\begin{tabular}{|l|l||}
\hline & Intervention \\
\hline A1 & $\begin{array}{l}\text { Systematic review of at least 2 independent from each other } \\
\text { conducted studies of evidence level A2 }\end{array}$ \\
\hline A2 & $\begin{array}{l}\text { Randomized double-blinded comparative clinical research of } \\
\text { good quality and efficient size }\end{array}$ \\
\hline B & $\begin{array}{l}\text { Comparative research, but not with all characteristics } \\
\text { as mentioned for A2. This includes also patient-control } \\
\text { research and cohort research }\end{array}$ \\
\hline C & Not comparative research \\
\hline D & Opinion of experts \\
\hline
\end{tabular}

study controlled for age, gender and pain duration, respectively. Also for the "Exposure dimension," Exposure 2 was added, since some studies had two outcome measures related to pain beliefs, cognitions, and behaviors. In order to allocate a star when assessing items 9 and 12 , the outcome instrument(s) should be validated for both populations. The maximum obtainable scores were 10 and 13 depending on the presence of 1 or 2 exposure(s), respectively. The explanations of all items are presented in Table 1.

In accordance with previous systematic reviews $(3,41)$, the 2005 classification system of the Dutch Institute for Healthcare Improvement was used to rate the level of evidence of each study based on the study design and risk of bias assessment (Table 2). Afterwards, the studies were clustered according to the outcome
Table 3. Strength of Conclusion (modified from www.cbo.nl).

\begin{tabular}{|l|l|}
\hline & Conclusion based on \\
\hline 1 & $\begin{array}{l}\text { Research of evidence level A1 or at least } 2 \text { independent } \\
\text { conducted studies of evidence level A2 }\end{array}$ \\
\hline 2 & $\begin{array}{l}1 \text { research of evidence level A2 or at least } 2 \text { independent } \\
\text { conducted studies of evidence level B }\end{array}$ \\
\hline 3 & 1 research of evidence level B or C \\
\hline 4 & $\begin{array}{l}\text { Opinion of experts or Inconclusive or inconsistent results } \\
\text { between various studies }\end{array}$ \\
\hline
\end{tabular}

measure and ethnic populations, and the strength of conclusion (ranging between 1 and 4) was determined for each of the clusters (Table 3 ).

\section{Data Extraction}

The data from the studies were manually synthesized and extracted by the first author and reported in an evidence table (Table 4) consisting of the following information:

1) Publication (author and year of publication];

2) Patients (sample size, the type of chronic MSKP, mean age, gender)

3) Outcome measurements (scales or questionnaires for pain beliefs, cognitions, and behaviors),

4) Main results (the differences between racial, ethnic, and cultural populations were presented by mean or standard deviation, and non-adjusted and eventual adjusted $P$ values); and

5) Study design. 


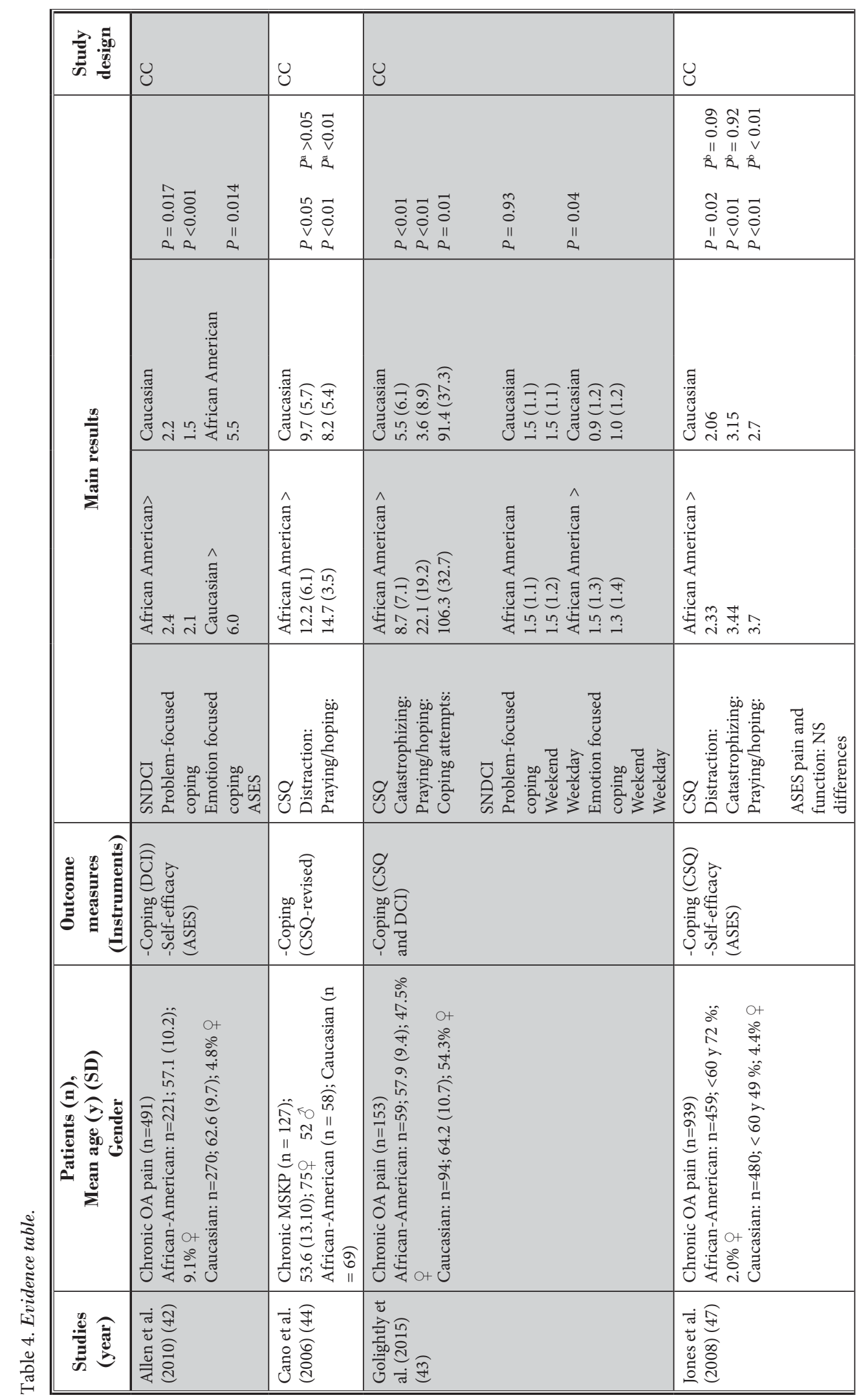




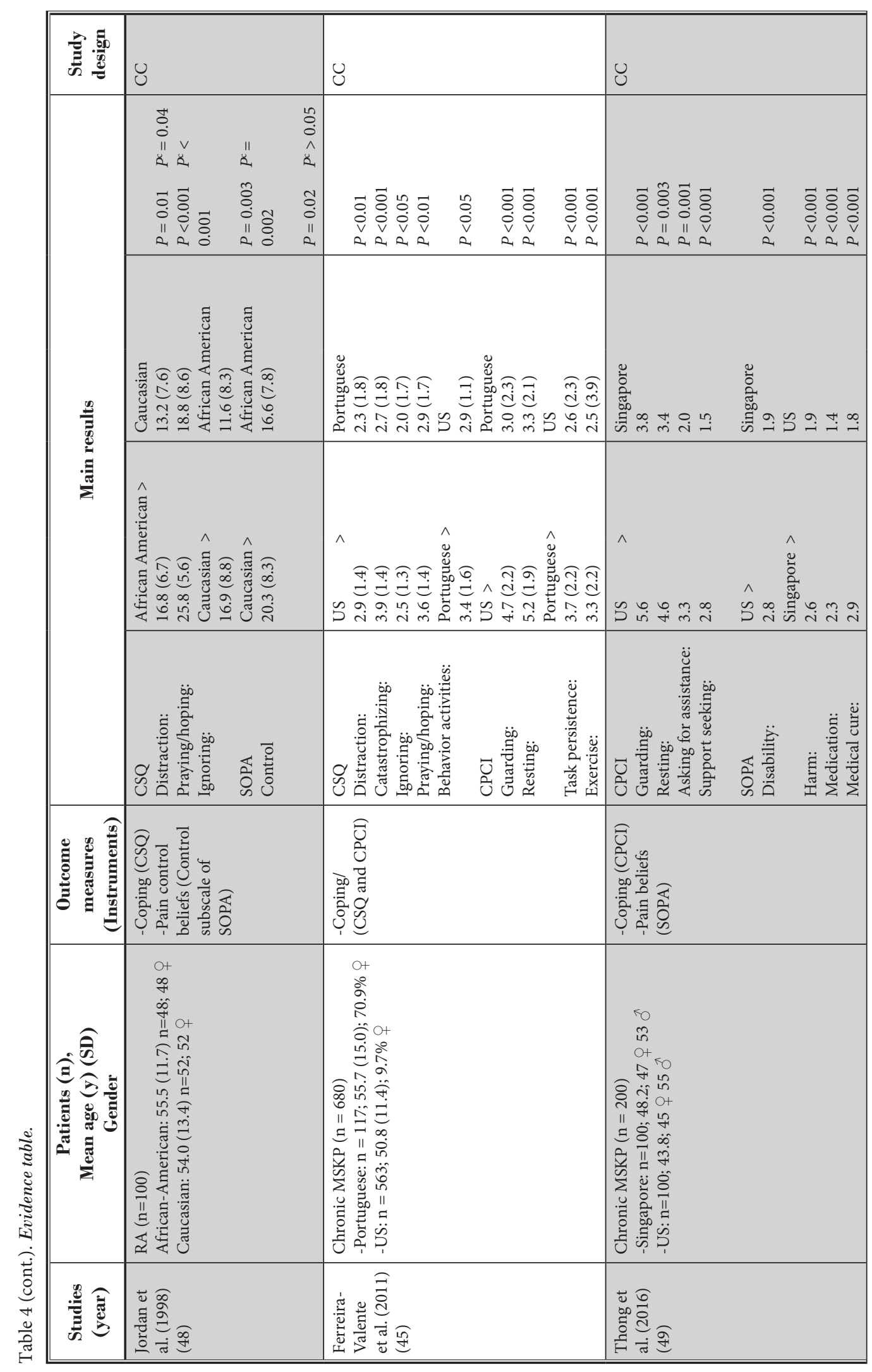




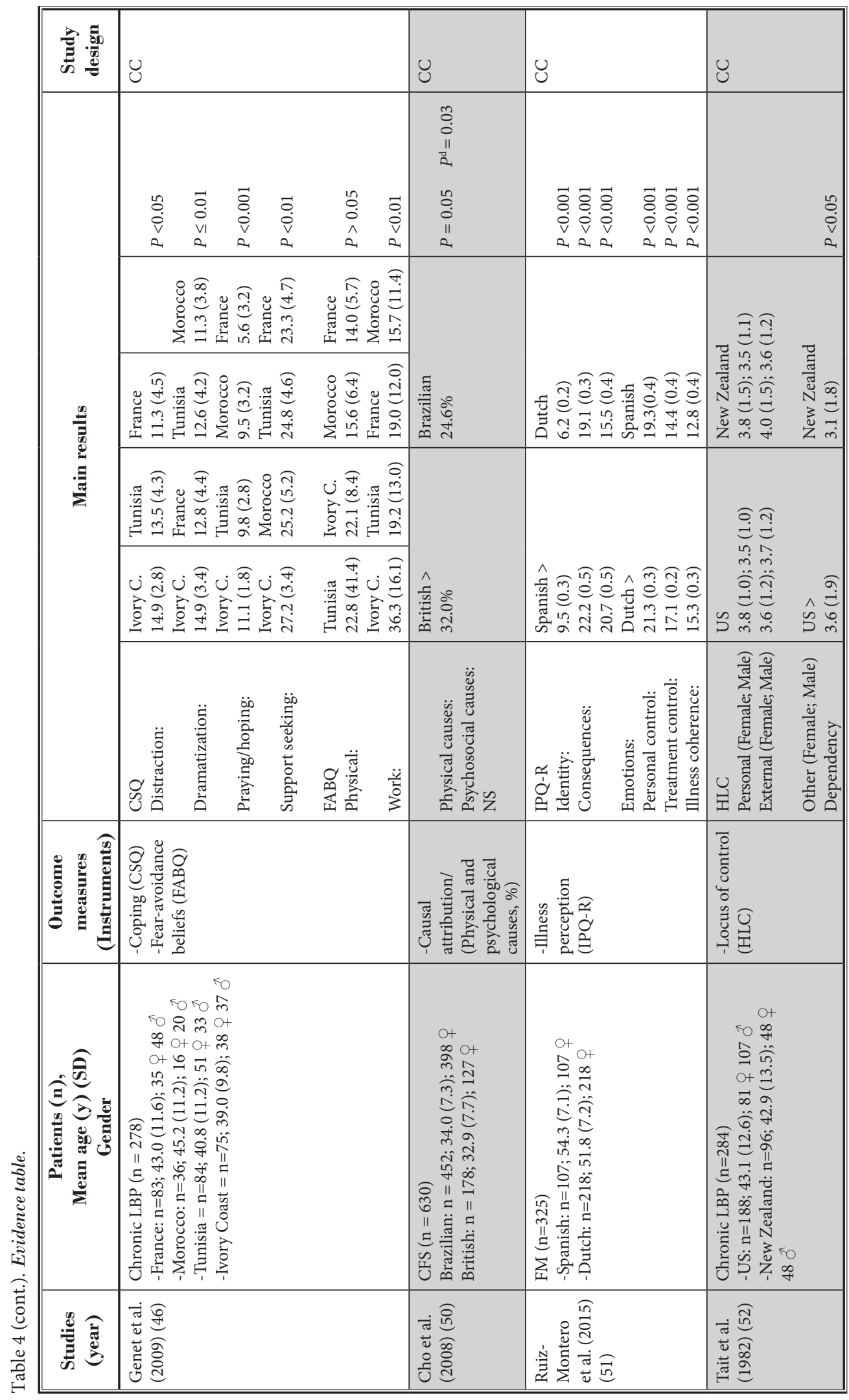

焉㺃害

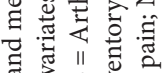

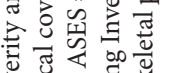

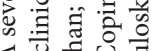

OS

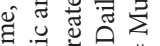

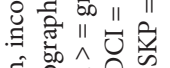

of

可

额

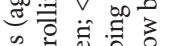

政

苍陆寻

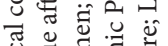

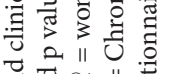

ज्ञ

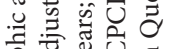

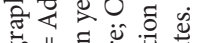

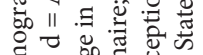

चั

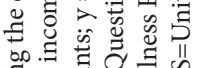

on.

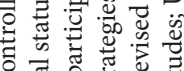

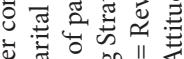

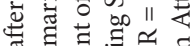

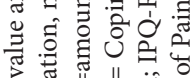

2. U્ّ

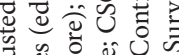

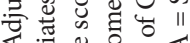

II 局总记

䓎

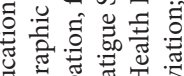

专

of

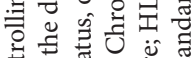

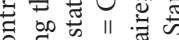

论

正

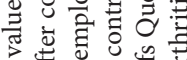

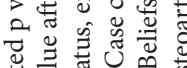

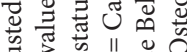

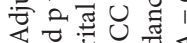

II 可

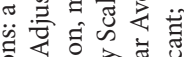

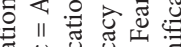

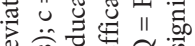

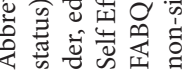




\section{Results}

\section{Study Selection}

The selection process of relevant articles is presented in the flow chart (Fig. 1). A total of 3,753 items were identified through the database search. After removing the duplicates, 3,320 studies were assessed during the first screening. Based on hand searching of reference lists, 2 additional articles $(42,43)$ were found. During the first screening, 34 studies were determined as potential eligible studies. Eleven full-text articles met the inclusion criteria after the second screening and were included to the qualitative synthesis of this systematic review.

\section{Study Characteristics}

All included articles $(n=11)$ were designed as a case control study comparing at least 2 different racial, ethnic, or cultural populations. The results of the studies were clustered into 2 categories based on pain related beliefs, cognitions, and behaviors, namely: 1 group of outcomes assessing pain coping (42-49) and 1 cluster of outcomes on perceptions and beliefs $(42,46-52)$.

The sample sizes of the included studies varied from 100 to 939 patients with chronic MSKP. Except for one study (47), in which the ages of participants were presented in percentages, the mean age of all patients was 47.2 years. In 2 studies $(48,51)$, only female patients

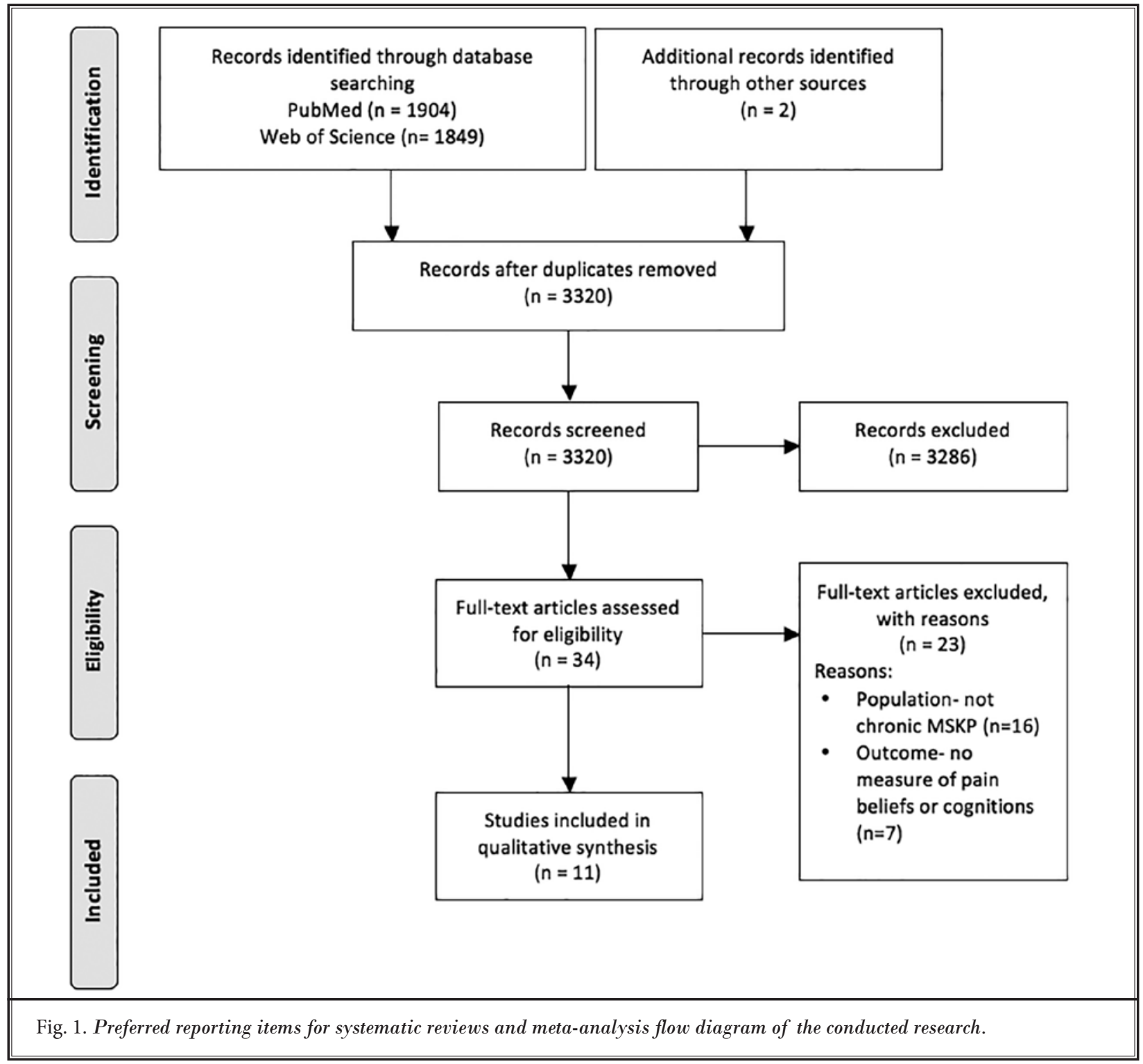


were recruited. In this systematic review, 8 studies included patients with specific diagnosis including chronic low back pain (LBP) $(46,52)$, osteoarthritis (OA) $(42,43,47)$, rheumatoid arthritis (RA) (48), chronic fatigue syndrome (CFS) (50), and fibromyalgia (FM) (51). Another 3 studies included mixed diagnosis of chronic $\operatorname{MSKP}(44,45,49)$ : in 1 study, most of the patients had a diagnosis of OA, or FM, or spine/disc problems (44); 1 study included patients with both back and neck pain (49); whereas 1 study did not indicate the types of chronic MSKP (45).

\section{Risk of Bias within Studies and Level of Evidence}

The scores and information regarding the risk of bias and level of evidence are presented in Table 1. An agreement was reached between both raters in most cases (83\%, 109 of 131 items). An additional discussion led to an agreement in 95\% (124 of 131 items) of the cases. Remaining differences ( 7 items) were solved by the third researcher (MM).

Methodological quality of the studies ranged from $20 \%$ to $54 \%$. Since all studies followed a comparative design, the level of evidence was $B$. The most common flaws for the studies were the definition of cases (100\%) (42-52), ascertainment of exposure (valid questionnaire for each population) (82\%) $(42,43,45-50,52)$, and representativeness of the cases $(73 \%)(42,43,45,46,48-50,52)$. No study provided information on birthplace, the country in which the person is living, and the parent's country of origin. Although Genet et al (46) did consider the place of birth and the country in which the person is living, this study did not receive stars for item 1 and 4 since information about the parent's country of origin was still lacking. Only 2 studies $(44,51)$ used valid questionnaires to evaluate the differences in pain coping and illness perceptions between different racial and cultural populations. In only 3 studies $(44,47,51)$, participants were recruited from a random sample in order to increase the generalization. The strengths of the studies were the use of the same instrument in order to evaluate pain beliefs, cognitions, and behaviors $(100 \%)(42-52)$ and the same non-response rate for both groups $(42,43,45-49,52)(73 \%)$.

\section{Synthesis of Results}

\section{Differences in the Coping Strategies}

Pain coping was investigated in 8 of 13 studies (4249). Five of them evaluated the discrepancies between
African-Americans and Caucasians $(42-44,47,48)$, another 3 articles examined the differences between other populations such as Portugal and United States (US) (45), Singapore, and the US (49), and 4 French speaking countries (46). Coping strategies were assessed by using the Coping Strategies Questionnaire (CSQ) in 6 studies (43-48), by Daily Coping Inventory (DCI) in 2 studies $(42,43)$, and by Chronic Pain Coping Inventory $(\mathrm{CPCl})$ in 2 studies $(45,49)$.

\section{African Americans and Caucasians}

Regarding the coping subscales of CSQ, 4 studies $(43,44,47,48)$ reported that African-Americans used the praying and hoping strategy more often than Caucasians. Three studies also presented adjusted $P$ values $(44,47,48)$. After controlling for socioeconomics and clinical covariates, there were still significant racial differences in the use of praying and hoping $(44,47,48)$. Three studies also found that African-Americans used more commonly "distraction" as a coping strategy than Caucasians $(44,47,48)$. Jordan et al $(48)$ showed that distraction strategy still remained significantly different between groups after performing regression analyses controlled for income, marital status, and education. However, 2 studies $(44,47)$ reported that there was no longer a significant difference in distraction between African-Americans and Caucasians after controlling for the demographic and clinical covariates. Although, Jones et al (47) reported that a trend towards a higher use of distraction was still present in African-Americans than in Caucasians.

Two studies found significant differences in the catastrophizing subscale of CSQ $(43,47)$. When compared to Caucasians, African-Americans used more catastrophizing. Jones et al (47) found that after adjusting for socio-economical and clinical covariates, the use of the catastrophizing strategy was similar between groups.

Golightly et al (43) also reported that AfricanAmericans attained higher number of coping attempts. Furthermore, Jordan et al (48) showed that Caucasians used more coping strategies involving ignoring pain. After performing regression analyses controlled for income, marital status, and education, the use of the ignoring pain strategy still remained significant between Caucasians and African-Americans (48).

In conclusion, there is moderate evidence that African-American patients with chronic MSKP use more praying and hoping, distraction, and catastrophizing strategies than Caucasians (strength of conclusion 2). 
However, when considering the adjusted $P$ values after controlling for socio-demographical and clinical covariates, there is only moderate evidence for praying and hoping (strength of conclusion 3), and there is preliminary evidence regarding the differences in distraction, catastrophizing, and coping attempts strategies between African-Americans and Caucasians (strength of conclusion 3). Additionally, there is preliminary evidence that Caucasians use more adaptive coping strategies involving ignoring pain (strength of conclusion 3).

Regarding the scores of the $\mathrm{DCl}$, Allen et al (42) and Golightly et al (43) found that African-Americans used more emotion-focused coping strategies than Caucasians. For the problem solving coping strategy, the difference between African-Americans and Caucasians was only observed in 1 study (42). Allen et al also showed that emotion focused coping is one of the key factors in the reduction of the racial differences in pain and function (42).

In conclusion, there is moderate evidence that African-American patients use more emotion-focused coping strategies than Caucasians (strength of conclusion 2). Additionally, there is preliminary evidence regarding the differences of problem solving coping strategies between African-Americans and Caucasians (strength of conclusion 3).

\section{Other populations}

Regarding the comparisons between US and Portuguese populations, significant differences were found in 9 of the 15 coping subscales (45). When considering the subscales of the CSQ, US patients used more distraction, catastrophizing, ignoring, praying and hoping strategies than Portuguese patients, whereas Portuguese patients more frequently used the increasing behavioural activities coping strategy. For the subscales of the $\mathrm{CPCl}$, the use of guarding and resting strategy was greater in US population than in Portuguese patients, while Portuguese patients used more frequently coping responses related to task persistence and exercise/ stretch than US patients.

Thong et al (49) reported that potential cultural differences can be seen between the patients from the US and those from Singapore. The US population obtained higher scores on the use of guarding, resting, asking for assistance, and support seeking than the patients from Singapore. In the Singapore population, guarding, resting, and relaxation subscales of the $\mathrm{CPCl}$ were positively and significantly associated with the pain intensity, whereas in the US population, they were negatively and non-significantly correlated with the pain intensity.

Genet et al (46) compared coping strategies among patients from four French-speaking countries. They revealed that patients living at the Ivory Coast used more distraction, dramatization, seeking social support, and praying and hoping strategies than Tunisian, French and Moroccan participants. However, the difference in distraction strategy between patients from Ivory Coast and Morocco was not significant. The strategy of ignoring was used most commonly in patients from Tunisia, although there were no significant differences between pairwise comparisons. In addition, the strategy of reinterpretation was more often used in patients from Tunisia than in patients from Morocco, the Ivory Coast or France; but only the comparison between Tunisian and French participants was significantly different $(P=0.02)$.

In conclusion, there is preliminary evidence that patients from the US use more coping strategies including distraction, catastrophizing, ignoring, praying and hoping, guarding and resting than patients from Portugal (strength of conclusion 3). There is also preliminary evidence that Portuguese patients have a more frequent use of increasing behavioural activities, task persistence and exercise/stretch than patients from the US (strength of conclusion 3). Additionally, there is preliminary evidence that patients from the US more frequently use coping strategies including guarding, resting, asking for assistance, and support seeking than Singaporean patients (strength of conclusion 3). Lastly, there is preliminary evidence that patients from the Ivory Coast use more coping strategies including distraction, dramatization, seeking social support strategies, and praying and hoping than patients from other French-speaking countries (strength of conclusion 3).

\section{Differences in the Illness Perceptions and Beliefs}

The differences in illness perceptions and control beliefs were investigated by seven studies $(42,47-52)$ : two articles investigated the discrepancies in self-efficacy between African-Americans and Caucasians (42,47); 2 of them compared the perceptions about diseases between Brazilian and British (50), and Spanish and Dutch patients (51); 2 articles reported the differences in pain-control beliefs between African-Americans and Caucasians (48), and patients from New Zealand and the US (52); 1 study investigated differences in fear avoidance beliefs among 4 French speaking countries (46), and a single study revealed the differences in pain attitudes and beliefs between the patients from US and Singapore (49). 


\section{Illness Perceptions}

Illness perceptions were evaluated by using the physical and psychological causes of fatigue (50) and Revised IIIness Perception Scale (51). One study revealed that when comparing the Brazilian CFS patients to British patients, that the latter reported their fatigue as being more likely associated with physical causes (50). Cho et al (50) revealed after controlling for socio-demographical and clinical covariates (such as gender, education, marital status, employment status, occupation, and fatigue score), that physical attribution remained significantly associated with country. Ruiz-Montero et al (51) found that Spanish females had more negative perceptions (identity, consequences, and emotions) than Dutch females. Consistently, Dutch patients showed a more positive understanding of the disease and showed at the same time more positive beliefs about the controllability of the disease. In both populations, the subscales related to the negative perceptions about disease were positively correlated with the FM impact, whereas positive perceptions such as personal and treatment control were negatively correlated with the FM impact (51).

In conclusion, there is preliminary evidence regarding the differences in physical attribution between British and Brazilian CFS patients (strength of conclusion 3). In addition, there is preliminary evidence that Spanish patients with FM have more negative perceptions than Dutch patients, and that Dutch patients have a more positive understanding about the disease and that they have more positive beliefs about the controllability of the disease (strength of conclusion 3).

\section{Self-Efficacy}

Self-efficacy was evaluated in 2 studies with the use of the Arthritis Self-Efficacy Scale (ASES) $(42,47)$. Conflicting results were presented regarding self-efficacy. While Allen et al (42) found that Caucasians had higher scores on ASES than African-Americans; Jones et al (47) demonstrated that African-Americans and Caucasians had similar self-efficacy scores. Allen et al (42) also indicated that lower self-efficacy was associated with worse pain and function, and it was demonstrated that self-efficacy is an important factor to reduce the racial differences in pain and function in patients with $O A$.

In conclusion, there is preliminary evidence that Caucasians have higher self-efficacy than AfricanAmericans (strength of conclusion 3).

\section{Fear Avoidance Beliefs}

One study (46) investigated the differences in fear avoidance beliefs using the Fear Avoidance Beliefs Questionnaire (FABQ) among 4 French-speaking countries (46). Fear and avoidance related to work was higher in patients from the Ivory Coast than in patients from Tunisia, France and Morocco. The differences between the patients from Ivory Coast and patients from other countries were very large. In addition, the score in the area of avoidance related to physical activities was the highest in patients from Tunisia when compared to the patients from the Ivory Coast, Morocco and France.

In conclusion, there is preliminary evidence that patients from Morocco and France have less fear avoidance beliefs regarding physical activities and work (strength of conclusion 3).

\section{Locus of Control}

Another study (52) used the Health Locus of Control survey to find differences in locus of control between New Zealand and US patients. New Zealanders had more internal health expectancies resulting in less dependency on physicians than US patients, whereas personal and external control were similar between the populations.

In conclusion, there is preliminary evidence that New Zealanders have more internal health expectancies than patients from the US (strength of conclusion 3).

\section{Pain Attitudes and Beliefs}

One study (48) investigated the differences in paincontrol beliefs between African-Americans and Caucasians using the pain control scale of the Survey of Pain Attitudes (SOPA). It was found that Caucasians with RA had more positive beliefs regarding the ability to control pain (48). However, there were no differences between African-Americans and Caucasians in the results of the regression analyses when controlled for pain beliefs pertaining to income, marital status, and education. Only one study (46) examined the differences in pain-related beliefs and attitudes between patients from the US and Singapore by using the SOPA. Patients from the US had higher disability subscale scores than patients from Singapore. However, patients from Singapore achieved greater scores in pain beliefs associated with the medical perspective on such as itmes the relation between damage and pain, pain medications and medical cure for chronic pain (49).

In conclusion, there is preliminary evidence that Caucasians with RA had more positive control beliefs than African-Americans (strength of conclusion 3) 
before adjusting for sociodemographics covariates. Additionally, there is preliminary evidence that patients from the US believe that they are more disabled, while Singaporeans interpret the pain more by a traditional biomedical perspective (strength of conclusion 3 ).

\section{Discussion}

The objective of the present study was to systematically review the current evidence regarding the racial, ethnic, and cultural differences in pain beliefs, cognitions, and behaviors of patients with chronic MSKP. The included studies examined the racial, ethnic, and cultural differences in pain-related beliefs, cognitions, and behaviors such as coping, illness perceptions, self-efficacy, fear avoidance, locus of control, and pain attitudes in patients with chronic MSKP. Overall, there is preliminary to moderate evidence regarding the differences in pain coping strategies, illness perceptions, self-efficacy, fear avoidance beliefs, locus of control, and pain attitudes of chronic MSKP patients among different races, ethnicities or cultures; however, the substantial risk of bias; the use of different scales to measure pain beliefs, cognitions, and behaviors; the comparisons of various populations; and the inconsistent controlling for confounding variables complicated the conclusions. To give an overview, all conclusions were summarized in Table 5.

To our knowledge, this is the first systematic review

Table 5. Summary of evidence regarding the differences in pain beliefs, cognitions, and behaviors between different races, ethnic populations and cultures.

\begin{tabular}{|c|c|c|c|c|}
\hline $\begin{array}{l}\text { Differences in pain beliefs, } \\
\text { cognitions, and behaviors }\end{array}$ & Ethnic Populations/ Cultures & $\begin{array}{l}\text { Strength of } \\
\text { conclusion }\end{array}$ & $\begin{array}{l}\text { Strength of } \\
\text { conclusion* }\end{array}$ & Reference \\
\hline $\begin{array}{l}\text { Pain coping } \\
\text {-Praying and hoping: } \\
\text {-Distraction: } \\
\text {-Catastrophizing: } \\
\text {-Ignoring pain: } \\
\text {-Coping attempts: } \\
\text {-Emotion focused coping: } \\
\text {-Problem focused coping: } \\
\text {-Distraction, catastrophizing, } \\
\text { ignoring praying and hoping, } \\
\text { guarding and resting } \\
\text {-Increasing behavioral } \\
\text { activities, task persistence, } \\
\text { exercise/stretch } \\
\text {-Guarding, resting, asking for } \\
\text { assistance, support seeking } \\
\text {-Distraction, dramatization, } \\
\text { seeking social support, praying } \\
\text { and hoping }\end{array}$ & $\begin{array}{l}\text { African-Americans }>\text { Caucasians } \\
\text { African-Americans }>\text { Caucasians } \\
\text { African-Americans }>\text { Caucasians } \\
\text { Caucasians }>\text { African-Americans } \\
\text { African-Americans }>\text { Caucasians } \\
\text { African-Americans }>\text { Caucasians } \\
\text { African-Americans }>\text { Caucasians } \\
\text { US }>\text { Portugal } \\
\text { Portugal }>\text { US } \\
\text { US }>\text { Singapore } \\
\text { Ivory Coast }>\text { Tunisia, Morocco, } \\
\text { France }\end{array}$ & $\begin{array}{l}\text { Moderate evidence (2) } \\
\text { Moderate evidence (2) } \\
\text { Moderate evidence (2) } \\
\text { Preliminary evidence (3) } \\
\text { Preliminary evidence (3) } \\
\text { Moderate evidence (2) } \\
\text { Preliminary evidence (3) } \\
\text { Preliminary evidence (3) } \\
\text { Preliminary evidence (3) } \\
\text { Preliminary evidence (3) } \\
\text { Preliminary evidence (3) }\end{array}$ & $\begin{array}{l}\text { Moderate evidence (2) } \\
\text { Preliminary evidence (3) } \\
\text { Preliminary evidence (3) } \\
\text { Preliminary evidence (3) } \\
\text { NA } \\
\text { NA } \\
\text { NA } \\
\text { NA } \\
\text { NA } \\
\text { NA } \\
\text { NA }\end{array}$ & $\begin{array}{l}(43,44,47,48) \\
(44,47,48) \\
(43,47) \\
(48) \\
(43) \\
(42,43) \\
(42,43) \\
(45) \\
(45) \\
(49) \\
(46)\end{array}$ \\
\hline $\begin{array}{l}\text { Illness perceptions } \\
\text {-Physical attribution } \\
\text {-Identity, consequences, } \\
\text { emotions } \\
\text {-Personal control, treatment } \\
\text { control, illness coherence }\end{array}$ & $\begin{array}{l}\text { British }>\text { Brazilian } \\
\text { Spanish }>\text { Dutch } \\
\text { Dutch }>\text { Spanish }\end{array}$ & $\begin{array}{l}\text { Preliminary evidence (3) } \\
\text { Preliminary evidence (3) } \\
\text { Preliminary evidence (3) }\end{array}$ & $\begin{array}{l}\text { Preliminary evidence (3) } \\
\text { NA } \\
\text { NA }\end{array}$ & $\begin{array}{l}(50) \\
(51) \\
(51)\end{array}$ \\
\hline Self-efficacy & Caucasians > African-Americans; & Preliminary evidence (3) & $\mathrm{NA}$ & $(42)$ \\
\hline Fear-avoidance beliefs & $\begin{array}{l}\text { Morocco \& France < Ivory Coast } \\
\text { \&Tunisia }\end{array}$ & Preliminary evidence (3) & NA & $(46)$ \\
\hline $\begin{array}{l}\text { Locus of control } \\
\text {-Internal health expectancies }\end{array}$ & New Zealanders $>$ US & Preliminary evidence (3) & NA & (52) \\
\hline $\begin{array}{l}\text { Pain attitudes and beliefs } \\
\text {-Ability to control pain } \\
\text {-Disability } \\
\text {-Harm, medication, medical cure }\end{array}$ & $\begin{array}{l}\text { Caucasians }>\text { African-Americans } \\
\text { US }>\text { Singapore } \\
\text { Singapore }>\text { US }\end{array}$ & $\begin{array}{l}\text { Preliminary evidence (3) } \\
\text { Preliminary evidence (3) } \\
\text { Preliminary evidence (3) }\end{array}$ & $\begin{array}{l}\text { No evidence } \\
\text { NA } \\
\text { NA } \\
\end{array}$ & $\begin{array}{l}(48) \\
(49) \\
(49)\end{array}$ \\
\hline
\end{tabular}

${ }^{*}=$ Based on the adjusted P value after controlling the demographic and clinical covariates; $<=$ less than; $>=$ greater than; NA = Not applicable. 
that shows the evidence regarding the racial, ethnic, and cultural differences in pain beliefs, cognitions, and behaviors of patients with chronic MSKP. Based on the results of the present study, African-Americans used praying and hoping, distraction, catastrophizing, coping attempts, emotion, and problem-focused coping, more frequently than Caucasian patients. However, Caucasians engaged ignoring strategies more frequently than African-Americans. A previous meta-analysis (35) investigating the racial differences in pain coping between African-Americans and Caucasians revealed the similar results. Additionally, this meta-analysis also presented that Caucasians had greater task persistence strategies (35). The results of previous meta-analyses are related to both healthy individuals and patients with chronic pain. Therefore, it is difficult to compare these results with the findings of our present study, which only includes patients with chronic MSKP.

It has been reported that coping is defined as the use of cognitive and behavioural techniques, grouped into 3 most common categories as follows: cognitive versus behavioural strategies; active versus passive strategies; and problem-focused versus emotion focused strategies (35). Subscales of CSQ include cognitive (praying and hoping, distraction, catastrophizing, ignoring pain, reinterpreting pain, and coping selfstatements) and behavioural (increasing behavioural activities) coping strategies. "Distraction" strategy is sometimes named as a "diverting attention" (35). Additionally, coping strategies can be active behaviors to obtain information to solve pain-related problems, or these strategies can be passive including seeking help from other people (11). Lastly, coping strategies can be classified as emotion- or problem-focused strategies (35). Problem-focused coping targets the causes of pain and involves direct attempts; however, emotionfocused coping involves trying to control emotional reactions associated with pain (35).

Praying and hoping strategy is considered as a passive and maladaptive coping strategy, which is negatively associated with the level of activity (53). Similarly, in this review, one study (44) found that praying and hoping was positively associated with the pain intensity, physical, and psychological disability. Also, another study (48) revealed that praying and hoping was positively correlated with the inactivity level in African-American patients. However, previous studies reported that if a population is more religious, it is expected that they will pray when they live in a painful situation $(35,54)$. Therefore, when considering the cul- tural aspects of African-Americans, one could surmise that they pray more frequently than Caucasians. On the other hand, in some cultures (45), people may perceive the pain as a punishment of their sins. Therefore, people suffering from severe pain may have a tendency toward praying and hoping to deal with the negative interpretation of pain. Additionally, seeking social support and relaxation are considered as an adaptive coping strategies (53). However, it has been concluded that these strategies are multidimensional and that there is a lack of evidence for arguing whether they are adaptive or maladaptive. Additionally, when patients have greater disabilities, they tend to seek social support more frequently (55). These findings support that the "adaptive" or "maladaptive" character of coping strategies might differ among various races, ethnicities, or cultures.

In this systematic review, some cultures including African Americans, patients from the Ivory Coast, and the US used coping strategies more frequently. It has been reported that having more coping strategies is to be preferred over having fewer (35). However, this situation varied depending on the type of coping strategy. In the present systematic review, one study (48) found an interaction between the coping strategy of reinterpreting pain and the intensity of pain. While there was a positive correlation between reinterpreting pain and pain intensity for African-Americans, the correlation was negative for Caucasians. In the literature, it has also been reported that the greater use of coping strategies in African-Americans might be associated with increased pain intensity and decreased functional level, since previous studies revealed that African-Americans had worse pain and poorer function than Caucasians $(43,56,57)$.

There is also preliminary evidence regarding the differences in illness perceptions, self-efficacy, fear-avoidance beliefs, pain control beliefs, and pain attitudes among various racial, ethnic, or cultural populations. The differences between Brazilian and British patients in beliefs regarding the physical causes for their fatigue revealed that British patients have a more medical view and awareness of CFS than Brazilian patients (50). Dutch patients had more positive understanding about the disease and beliefs about controllability of the disease. Since the Dutch patients have lived longer with FM pain than Spanish patients, they might have developed coping strategies and acceptance for the symptoms of FM (51). One study found that self-efficacy is one of the most important factors 
that reduce the racial differences in pain and function in patients with OA (42). A recent systematic review (58) also suggested that higher self-efficacy levels were associated with greater physical functioning, participation in physical activities, work performance, and health status. Interestingly, one study (49) showed that patients from Singapore see themselves less disabled by the pain when compared to the US population. In Asian cultures, the ability to tolerate pain may be related to the stoicism which is sign of power and positive trait (49). Previous research has reported that stoicism is also associated with low treatment adherences and avoiding pain treatments $(59,60)$.

In the literature, self-efficacy, internal locus of control, adaptive pain coping strategies, and acceptance are suggested to be positive beliefs, which are associated with the improved adjustment of pain and also have an important impact on the prognosis of pain $(14,58,61)$. Self-efficacy is associated with the higher level of self-control defined as a personal confidence which is required for successfully achieving a desired outcome (58). Self-efficacy develops motivation, psychological well-being, and personal achievement (58). Locus of control term refers to the process of the control-over-reinforcements attribution, which can be internal or external (62). Patients who have internal locus of control perceive themselves as controlling events by individually, while those have external expectancies believe that these events can be controlled by some factors such as fate, luck, or powerful people (52). Acceptance related to chronic pain is defined as an active willingness to engage in meaningful activities despite pain-related sensations, thoughts or feelings (14). On the other hand, catastrophizing and high fear avoidance beliefs were associated with the poor treatment outcomes, higher pain, disability level, and work absenteeism $(14,63,64)$. Catastrophizing is a cognitive process that can affect perceptions, expectations, experiences, and memories by interpreting even minor problems as major situations (11). Negative beliefs about disease and pain cause catastrophizing responses, which are possible reasons for fear of activity and avoidance that lead to disuse or a decrease in activity level (64). Based on the fear-avoidance model, patients without fear-avoidance beliefs are more likely to overcome pain problems and use more adaptive coping strategies.

Recent systematic reviews have revealed the importance of pain beliefs and cognitions in the prognosis of chronic MSKP (58) and adherence to multidisciplinary rehabilitation (12). Therefore, the differences in pain beliefs, cognitions, and behaviors between races, ethnicities or cultures, and the impact of positive pain-related beliefs, cognitions, and behaviors on improved pain adjustment and treatment outcomes highlight that culturally sensitive adaptations of pain neuroscience education, psychological, and behavioural interventions such as cognitive behavioural therapy and mindfulness based therapy may be important to reduce the racial, ethnic, and cultural disparities in pain-related beliefs, cognitions, and behaviors and to improve the treatment outcomes. Literature has also suggested that strategies for designing the culturally sensitive interventions should focus on the cultural practices, philosophies, products, or environments $(26,65,66)$.

\section{Limitations and Strengths}

The present study had some limitations. The first limitation was that only 11 articles were identified for this systematic review. A second limitation lies in the methodological quality of the included studies, which ranged from low to moderate, and which had low levels of evidence since all of them were designed as case control studies. Additionally, pain-related beliefs, cognitions, and behaviors were reported by using different scales and questionnaires. Therefore, clustering the results, interpreting the findings, and consequently stating clear conclusions were limited due to the small numbers of articles, wide range of assessment methods, and low methodological quality. Lastly, it should be noted that studies $(45,46,50-52)$, which investigated the origin of country effect on pain beliefs, cognitions, and behaviors did not provide information about intraethnic variability, thus study populations might not be homogenous. Similarly, since it has been reported that a non-Hispanic, white population is hardly homogenous (26), the intra-ethnic variability should also be an important consideration for studies determining the differences between African-Americans and Caucasians $(42-44,47,48)$.

However, this systematic review also has several strengths. To our knowledge, this is the first overview investigating the influence of race, ethnicity, and culture on pain-related beliefs, cognitions, and behaviors in patients with chronic MSKP. Additionally, screening and bias analysis were performed by 2 independent and blinded researchers. Lastly, NOS was modified by adding an extra item for the comparability dimension and a second exposure category. Moreover, items related to the definitions of cases and ascertainments of 
exposures were specifically rated regarding the topic of this systematic review.

\section{Recommendations for Further Research}

According to the results of the present review, a clear gap regarding the racial, ethnic, and cultural differences in pain beliefs, cognitions, and behaviors should be filled. Therefore, the following recommendations are given to investigate the differences in pain-related beliefs, cognitions, and behaviors between different populations. First, there is a need for standard definitions to recruit patients based on race, ethnicity or culture. In most included studies, the definitions regarding race, ethnicity, or culture were self-reported or only country-based. We suggest to determine race, ethnicity, or culture based on the information regarding the birthplace, country of living, and the parent's country of origin. Furthermore, intra-ethnic variations should be considered in further research. For migrants and ethnic minorities, the individual's country of origin, migration status, years lived as a migrant, being a first or second generation migrant, and acculturation or assimilation should be taken into account (26).

In the included articles, pain beliefs, cognitions, and behaviors have been (retrospectively) evaluated by self-reported questionnaires on a cross-sectional basis. Only 2 studies $(42,43)$ used the $\mathrm{DCl}$ to evaluate the coping strategies prospectively during 1 week. It has been reported that cross-sectional measures may have an inability to capture important day-to-day variations (14). In the literature, it has been emphasized that coping diaries are important to better detect the dynamic process of coping and to predict the effects of coping strategies on pain and mood during the next day (14). Therefore, to explore the racial, ethnic, or cultural discrepancies in pain beliefs and cognitions, further prospective, and longitudinal studies are warranted. Additionally, it has been indicated that appropriate psychometric evaluation of scales including reliability and validity for each population have to be performed before the use of an instrument (11). One of the major concerns of the present review was that scales and questionnaires for measuring the pain related beliefs, cognitions, and behaviors were not indicated to be valid for each population.

Future research should also control the demographics and clinical covariates that may influence the pain beliefs, cognitions, and behaviors. In the present study, some studies controlled the covariates and inves- tigated the mediating effects of the demographic or clinical variables such as education, marital status, income, disease severity, and fatigue score $(44,47,48,50)$.

To date, studies revealed differences in pain coping, self-efficacy, and pain control beliefs between African-Americans and Caucasians. It could be interesting to study other pain-related beliefs such as catastrophizing, hypervigilance, acceptance, and somatization. Furthermore, there is no evidence regarding the racial, ethnic or cultural differences in pain beliefs and cognitions of other chronic MSKP disorders such as temporomandibular disorders, tension-type headache, chronic whiplash associated disorders, and chronic pelvic pain. Further investigation on these conditions is needed.

\section{Conclusion}

In conclusion, there is moderate evidence that African-Americans use more praying and hoping, and emotion-focused coping strategies than Caucasians. There is also preliminary evidence regarding the differences in some coping strategies such as distraction, catastrophizing, and problem-focused solving between African-Americans and Caucasians. Additionally, preliminary evidence exists regarding the differences in pain coping strategies between the US and Portugal; the US and Singapore; and among the 4 French-speaking countries. It is found that Spanish patients with FM have more negative illness perceptions than Dutch patients. There is preliminary evidence that Caucasians have higher self-efficacy than African-Americans. Additionally, patients from Morocco and France have less fear avoidance beliefs regarding physical activities and work. There is also preliminary evidence that New Zealanders have more internal health expectancies than patients from the US. Preliminary evidence is demonstrated that Caucasians with RA have more positive control beliefs than African-Americans. Lastly, there is preliminary evidence that patients from the US believe that they are more disabled, while Singaporeans interpret the pain more by a traditional biomedical perspective. The findings of this systematic review reveal that little is known about the racial, ethnic, or cultural discrepancies in pain beliefs, cognitions, and behaviors of patients with chronic MSKP. Further prospective and longitudinal studies using standard definitions for race, ethnicity, or culture, and valid questionnaires for each population are warranted to explore the racial, ethnic or cultural discrepancies in pain beliefs, cognitions, and behaviors. 


\section{Conflict Of Interests}

Authors declare that they have no conflicts of interests.

Disclaimer: Lenoir and Meeus contributed equally to this manuscript. The first author (CO) was financially supported by the Scientific Researches Department of Hacettepe University during the study period in Ghent University (Project number: TBI-2017-13866).

Conflict of interest: Each author certifies that he or she, or a member of his or her immediate family has no commercial association (i.e., consultancies, stock ownership, equity interest, patent/licensing arrangements, etc.) that might pose conflict of interest in connection with the submitted manuscript.

Author's contributions: Each of the authors has participated in this systematic review and has agreed to be an author on the paper. All the authors have read the manuscript and accepted submission. Authors meet the appropriate authorship criteria. All authors conducted the review, drafted the paper and revised it critically and checked the final version. DL and MM contributed equally to this manuscript.

\section{Acknowledgments}

The first author would like to express her gratitude to the Scientific Researches Department of Hacettepe University for financially supporting her during the study period in Ghent University (Project number: TBI-2017-13866).

\section{REFERENCES}

1. Treede RD, RiefW, Barke A, Aziz Q, Bennett MI, Benoliel R, Cohen M, Evers S, Finnerup NB, First MB, Giamberardino MA, Kaasa S, Kosek E, Lavand'homme P, Nicholas M, Perrot S, Scholz J, Schug S, Smith BH, Svensson P, Vlaeyen JW, Wang SJ. A classification of chronic pain for ICD-11. Pain 2015; 156:1003-1007.

2. Hague M, Shenker N. How to investigate: Chronic pain. Best Pract Res Clin Rheumatol 2014; 28:860-874.

3. Coppieters I, Meeus M, Kregel J, Caeyenberghs K, De Pauw R, Goubert D, Cagnie B. Relations between brain alterations and clinical pain measures in chronic musculoskeletal pain: aAsystematic review. J Pain 2016; 17:949-962.

4. Jordan JL, Holden MA, Mason EE, Foster NE. Interventions to improve adherence to exercise for chronic musculoskeletal pain in adults. Cochrane Database Syst Rev 2010:CDo05956.

5. McBeth J, Jones K. Epidemiology of chronic musculoskeletal pain. Best Pract Res Clin Rheumatol 2007; 21:403-425.

6. Breivik H, Collett B, Ventafridda V, Cohen R, Gallacher D. Survey of chronic pain in Europe: prevalence, impact on daily life, and treatment. Eur ] Pain 2006; 10:287-333.

7. Cimmino MA, Ferrone C, Cutolo M. Epidemiology of chronic musculoskeletal pain. Best Pract Res Clin Rheumatol 2011; 25:173-183.

8. Crofford LJ. Psychological aspects of chronic musculoskeletal pain. Best Pract
Res Clin Rheumatol 2015; 29:147-155.

9. Gatchel RJ, Peng YB, Peters ML, Fuchs PN, Turk DC. The biopsychosocial approach to chronic pain: Scientific advances and future directions. Psychol Bull 2007; 133:581-624.

10. Manchikanti L, Fellows B, Singh V. Understanding psychological aspects of chronic pain in interventional pain management. Pain Physician 2002; 5:57-82.

11. Turk DC, Fillingim RB, Ohrbach R, Patel KV. Assessment of psychosocial and functional impact of chronic pain. J Pain 2016; 17:T21-49.

12. Thompson EL, Broadbent J, Bertino MD, Staiger PK. Do Pain-related beliefs influence adherence to multidisciplinary rehabilitation?: A systematic review. Clin J Pain 2016; 32:164-178.

13. Main CJ, Foster N, Buchbinder R. How important are back pain beliefs and expectations for satisfactory recovery from back pain? Best Pract Res Clin Rheumatol 2010; 24:205-217.

14. Keefe FJ, Rumble ME, Scipio CD, Giordano LA, Perri LM. Psychological aspects of persistent pain: Current state of the science. J Pain 2004; 5:195-211.

15. Crombez G, Vlaeyen JW, Heuts PH, Lysens R. Pain-related fear is more disabling than pain itself: Evidence on the role of pain-related fear in chronic back pain disability. Pain 1999; 80:329-339.

16. Thomas E, Silman AJ, Croft PR, Papageorgiou AC, Jayson MI, Macfarlane GJ.
Predicting who develops chronic low back pain in primary care: A prospective study. BMJ 1999; 318:1662-1667.

17. Stroud MW, Thorn BE, Jensen MP, Boothby JL. The relation between pain beliefs, negative thoughts, and psychosocial functioning in chronic pain patients. Pain 2000; 84:347-352.

18. Thibault P, Loisel P, Durand MJ, Catchlove R, Sullivan MJ. Psychological predictors of pain expression and activity intolerance in chronic pain patients. Pain 2008; 139:47-54.

19. Williams DA, Keefe FJ. Pain beliefs and the use of cognitive-behavioral coping strategies. Pain 1991; 46:185-190.

20. Williams DA, Thorn BE. An empirical assessment of pain beliefs. Pain 1989; 36:351-358.

21. Nieto R, Raichle KA, Jensen MP, Miro J. Changes in pain-related beliefs, coping, and catastrophizing predict changes in pain intensity, pain interference, and psychological functioning in individuals with myotonic muscular dystrophy and facioscapulohumeral dystrophy. Clin J Pain 2012; 28:47-54.

22. Jensen MP, Turner JA, Romano JM. Changes in beliefs, catastrophizing, and coping are associated with improvement in multidisciplinary pain treatment. J Consult Clin Psychol 2001; 69:655-662.

23. Jensen MP, Turner JA, Romano JM. Changes after multidisciplinary pain treatment in patient pain beliefs and coping are associated with concurrent 
changes in patient functioning. Pain 2007; 131:38-47.

24. Lasch KE. Culture, pain, and culturally sensitive pain care. Pain Manag Nurs 2000; 1:16-22.

25. Campbell CM, Edwards RR. Ethnic differences in pain and pain management. Pain Manag 2012; 2:219-230.

26. Anderson KO, Green CR, Payne R. Racial and ethnic disparities in pain: Causes and consequences of unequal care. J Pain 2009; 10:1187-1204.

27. Cintron A, Morrison RS. Pain and ethnicity in the United States: A systematic review. J Palliat Med 2006; 9:1454-1473.

28. Edwards CL, Fillingim RB, Keefe F. Race, ethnicity and pain. Pain 2001; 94:133-137.

29. Green CR, Anderson KO, Baker TA, Campbell LC, Decker S, Fillingim RB, Kalauokalani DA, Lasch KE, Myers C, Tait RC, Todd KH, Vallerand AH. The unequal burden of pain: Confronting racial and ethnic disparities in pain. Pain Med 2003; 4:277-294.

30. Rahim-Williams B, Riley JL, 3rd, Williams AK, Fillingim RB. A quantitative review of ethnic group differences in experimental pain response: Do biology, psychology, and culture matter? Pain Med 2012; 13:522-540.

31. Ezenwa MO, Ameringer S, Ward SE, Serlin RC. Racial and ethnic disparities in pain management in the United States. J Nurs Scholarsh 2006; 38:225-233.

32. Al-Harthy M, Ohrbach R, Michelotti A, List T. The effect of culture on pain sensitivity. J Oral Rehabil 2016; 43:81-88.

33. Henschke N, Lorenz E, Pokora R, Michaleff ZA, Quartey JNA, Oliveira VC. Understanding cultural influences on back pain and back pain research. Best Pract Res Clin Rheumatol 2016; 30:1037-1049.

34. Njobvu P, Hunt I, Pope D, Macfarlane G. Pain amongst ethnic minority groups of South Asian origin in the United Kingdom: A review. Rheumatology (Oxford) 1999; 38:1184-1187.

35. Meints SM, Miller MM, Hirsh AT. Differences in pain coping between black and white americans: A meta-analysis. J Pain 2016; 17:642-653.

36. Moher D, Liberati A, Tetzlaff J, Altman DG, Group P. Preferred reporting items for systematic reviews and meta-analyses: The PRISMA statement. Int J Surg 2010; 8:336-341.

37. Ouzzani M, Hammady H, Fedorowicz Z,
Elmagarmid A. Rayyan-a web and mobile app for systematic reviews. Syst Rev 2016; 5:210.

38. Wells GA, Shea B, O'Connell D, Peterson J, Welch V, Losos M, Tugwell P. The Newcastle-Ottawa Scale (NOS) for assessing the quality of nonrandomised studies in meta-analyses. Avaliable at: http://www.ohri.ca/programs/clinical_ epidemiology/oxford.asp. Accessed at February 5, 2018.

39. Zeng X, Zhang Y, Kwong JS, Zhang C, Li S, Sun F, Niu Y, Du L. The methodological quality assessment tools for preclinical and clinical studies, systematic review and meta-analysis, and clinical practice guideline: A systematic review. J Evid Based Med 2015; 8:2-10.

40. Stang A. Critical evaluation of the Newcastle-Ottawa scale for the assessment of the quality of nonrandomized studies in meta-analyses. Eur J Epidemiol 2010; 25:603-605.

41. DePauw R, Coppieters I, Meeus M, Caeyenberghs K, Danneels L, Cagnie B. Is traumatic and non-traumatic neck pain associated with brain alterations? A systematic review. Pain Physician 2017; 20:245-26o.

42. Allen KD, Oddone EZ, Coffman CJ, Keefe FJ, Lindquist JH, Bosworth HB. Racial differences in osteoarthritis pain and function: Potential explanatory factors. Osteoarthritis Cartilage 2010; 18:160-167.

43. Golightly YM, Allen KD, Stechuchak KM, Coffman CJ, Keefe FJ. Associations of coping strategies with diary based pain variables among Caucasian and African American patients with osteoarthritis. Int J Behav Med 2015; 22:101-108.

44. Cano A, Mayo A, Ventimiglia M. Coping, pain severity, interference, and disability: The potential mediating and moderating roles of race and education.J Pain 2006; 7:459-468.

45. Ferreira-Valente MA, Ribeiro JL, Jensen MP, Almeida R. Coping with chronic musculoskeletal pain in Portugal and in the United States: A cross-cultural study. Pain Med 2011; 12:1470-1480.

46. Genet F, Autret K, Roche N, Lapeyre E, Schnitzler A, Mandjui B, Manou B, Dziri C, Helleuch H, Rejeb N, Oudghiri N, Revel M, Poiraudeau S. Comparison of the repercussions of CLBP in four French-speaking countries. Ann Phys Rehabil Med 2009; 52:717-728.

47. Jones AC, Kwoh CK, Groeneveld PW,
Mor M, Geng M, Ibrahim SA. Investigating racial differences in coping with chronic osteoarthritis pain. J Cross Cult Gerontol 2008; 23:339-347.

48. Jordan MS, Lumley MA, Leisen JC. The relationships of cognitive coping and pain control beliefs to pain and adjustment among African-American and Caucasian women with rheumatoid arthritis. Arthritis Care Res 1998; 11:80-88.

49. Thong ISK, Tan G, Lee TYC, Jensen MP. A comparison of pain beliefs and coping strategies and their association with chronic pain adjustment between Singapore and United States. Pain Med 2017; 18:1668-1678.

50. Cho HJ, Bhugra D, Wessely S. 'Physical or psychological?'- a comparative study of causal attribution for chronic fatigue in Brazilian and British primary care patients. Acta Psychiatr Scand 2008; 118:34-41.

51. Ruiz-Montero PJ, Van Wilgen CP, Segura-Jimenez V, Carbonell-Baeza A, Delgado-Fernandez M. Illness perception and fibromyalgia impact on female patients from Spain and the Netherlands: Do cultural differences exist? Rheumatol Int 2015; 35:1985-1993.

52. Tait R, DeGood D, Carron H. A comparison of health locus of control beliefs in low-back patients from the U.S. and New Zealand. Pain 1982; 14:53-61.

53. Monticone M, Ferrante S, Giorgi I, Galandra C, Rocca B, Foti C. The 27-item coping strategies questionnaire-revised: Confirmatory factor analysis, reliability and validity in Italian-speaking subjects with chronic pain. Pain Res Manag 2014; 19:153-158.

54. Chatters LM, Taylor RJ, Bullard KM, Jackson JS. Race and ethnic differences in religious involvement: African Americans, Caribbean Blacks and NonHispanic Whites. Ethn Racial Stud 2009; 32:1143-1163.

55. Tan G, Nguyen $Q$, Anderson KO, Jensen $M$, Thornby J. Further validation of the chronic pain coping inventory. J Pain 2005; 6:29-40.

56. Golightly YM, Dominick KL. Racial variations in self-reported osteoarthritis symptom severity among veterans. Aging Clin Exp Res 2005; 17:264-269.

57. Jordan JM, Luta G, Renner JB, Dragomir A, Hochberg MC, Fryer JG. Ethnic differences in self-reported functional status in the rural south: The Johnston County Osteoarthritis Project. Arthritis 
Care Res 1996; 9:483-491.

58. Martinez-Calderon J, Zamora-Campos C, Navarro-Ledesma S, Luque-Suarez A. The role of self-efficacy on the prognosis of chronic musculoskeletal pain: A systematic review. J Pain 2018; 19:10-34.

59. Juarez G, Ferrell B, Borneman T. Influence of culture on cancer pain management in Hispanic patients. Cancer Pract 1998; 6:262-269.

6o. Streltzer J, Wade TC. The influence of cultural group on the undertreatment of postoperative pain. Psychosom Med 1981; 43:397-403.

61. Burns JW, Glenn B, Bruehl S, Harden
RN, Lofland K. Cognitive factors influence outcome following multidisciplinary chronic pain treatment: A replication and extension of a cross-lagged panel analysis. Behav Res Ther 2003; 41:1163-1182.

62. Cano-Garcia FJ, Rodriguez-Franco L, Lopez-Jimenez AM. Locus of control patterns in headaches and chronic pain. Pain Res Manag 2013; 18:e48-54.

63. Geisser ME, Robinson ME, Riley JL. Pain beliefs, coping, and adjustment to chronic pain - Let's focus more on the negative. Pain Forum 1999; 8:161-168.

64. Wertli MM, Rasmussen-Barr E, Held
U, Weiser S, Bachmann LM, Brunner F. Fear-avoidance beliefs- a moderator of treatment efficacy in patients with low back pain: A systematic review. Spine J 2014; 14:2658-2678.

65. Chin MH, Walters AE, Cook SC, Huang ES. Interventions to reduce racial and ethnic disparities in health care. Med Care Res Rev 2007; 64:7S-28S.

66. Fisher TL, Burnet DL, Huang ES, Chin $\mathrm{MH}$, Cagney KA. Cultural leverage: interventions using culture to narrow racial disparities in health care. Med Care Res Rev 2007; 64:243S-282S. 